\title{
Long-term Survival of Patient with Ampulla of Vater Metastasis of Renal Cell Carcinoma
}

\author{
Povilas Ignatavicius ${ }^{1}$, Paulius Lizdenis ${ }^{1}$, Darius Pranys ${ }^{2}$, \\ Antanas Gulbinas ${ }^{1}$, Juozas Pundzius ${ }^{1}$, Giedrius Barauskas $^{1}$ \\ ${ }^{1}$ Department of Surgery, Lithuanian University of Health Sciences, Kaunas, Lithuania; \\ ${ }^{2}$ Department of Pathology, Lithuanian University of Health Sciences, Kaunas, \\ Lithuania \\ Received October 1, 2018; Accepted January 31, 2019.
}

Key words: Renal cell carcinoma - Metastasis - Ampulla of Vater Pancreatoduodenectomy

\begin{abstract}
Ampulla of Vater metastases from renal cell carcinoma are rare. The time between detection of the primary tumour and its metastasis may extend to years. Management should be aggressive, since the prognosis of renal cell carcinoma is unpredictable and curative surgery of metastases may extend patient survival and even lead to definite cure. Herein we report a case of long-term survival after successful surgical treatment of a renal cell carcinoma metastasis to the ampulla of Vater. A 62-year-old man with a history of renal cell carcinoma in the left kidney underwent a successful left nephrectomy. Eight months later duodenoscopy showed a tumour at the site of papilla of Vater. Biopsy confirmed the diagnosis of carcinoma. Contrast enhanced computer tomography scan verified the periampullary mass, dilatation of the pancreatic and the common bile duct. No radiological signs of either local advancement or distant metastases were present. Pylorus-preserving pancreatoduodenectomy with lymphadenectomy was performed. Pathology report disclosed metastatic lesions in the papilla of Vater from the clear cell carcinoma of the kidney. The postoperative course was uneventful, and the patient lived for 14 years after pancreatoduodenectomy and, following thorough investigations, was free from local and systemic recurrence. Pancreatoduodenectomy can provide long-term survival in selected cases with solitary papilla of Vater metastasis from renal cell carcinoma. Favourable long-term survival rates suggest that these patients should be considered candidates for pancreatoduodenectomy if experienced pancreatic surgeon is available and no other metastases are found.
\end{abstract}

Mailing Address: Povilas Ignatavicius, MD., Department of Surgery, Lithuanian University of Health Sciences, Eiveniu Str. 2, 50009 Kaunas, Lithuania; Phone: +370 37326 751; Fax: +370 37327 163; e-mail: povilas.ignatavicius@Ismuni.It 


\section{Introduction}

Renal cell carcinoma is a neoplasm with unpredictable course even after metastasing (Pavlakis et al., 2004). Metastases from this type of cancer have been described from various organs, but ampulla of Vater metastases seem to be extremely rare, as only 12 cases have been reported in the literature. Even though there are several case reports of metastases to nearby pancreas (Hirota et al., 1996; Hashimoto et al., 2001; Wente et al., 2005; Karakatsanis et al., 2013; Haidong et al., 2014), and a case report of metastases both to the duodenum and the pancreas (Hashimoto et al., 2001), there are no obvious explanations for metastasis potential discrepancy in these sites.

Early stages of tumours metastasing to the ampulla of Vater usually give rise to few symptoms and signs, which make diagnosis difficult. Despite that, aggressive surgical treatment has been advocated for all solitary metastases arising from renal cell carcinoma, including ampullary metastases (Wente et al., 2005; Haidong et al., 2014). Discussing treatment options, it is important to bear in mind that patients with one metastasis may also have occult tumours in other sites (Merino et al., 2005). In this case report, we describe a patient with ampullary metastasis from renal cell carcinoma, presenting with abdominal pain. The diagnosis was established by means of endoscopy and biopsy and the patient has undergone successful surgery.

\section{Case report}

A 62-year-old man with a history of renal cell carcinoma in the left kidney underwent a successful left nephrectomy. Eight months later the patient was admitted to the Hospital of Lithuanian University of Health Sciences (Kaunas, Lithuania) with severe upper abdominal pain, jaundice, general weakness and subclinical signs of gastric outlet obstruction. Serum analysis revealed slight increase in total and direct bilirubin ( $64.8 \mu \mathrm{mol} / \mathrm{I}$ and $42.5 \mu \mathrm{mol} / \mathrm{I}$ respectively), whereas serum amylase was normal. Abdominal pain and jaundice have been described as transient during the course of his disease. Laboratory results were unspecific whereas duodenoscopy showed a tumour at the site of papilla of Vater. Biopsy confirmed the diagnosis of carcinoma. Contrast enhanced CT (computed tomography) scan verified the periampullary mass, slight dilatation of the pancreatic and the common bile duct. No radiological signs of either local advancement or distant metastases were present.

Standard pylorus-preserving pancreatoduodenectomy with regional lymphadenectomy was performed as no other manifestation of the malignancy was found. The underlying vascular structures were not involved by the tumour and the resection was classified as curative. Gross examination of the specimen revealed two tumours in the duodenum, one of them involving papilla of Vater and obstructing the orifice. Final pathology report disclosed the metastatic lesions in the duodenum and the papilla of Vater from the clear cell carcinoma of the kidney (Figure 1), also confirmed by immunohistochemical staining (Figure 2). Twenty lymph nodes in the surgical specimen were negative for metastatic lesions and the resection margins 

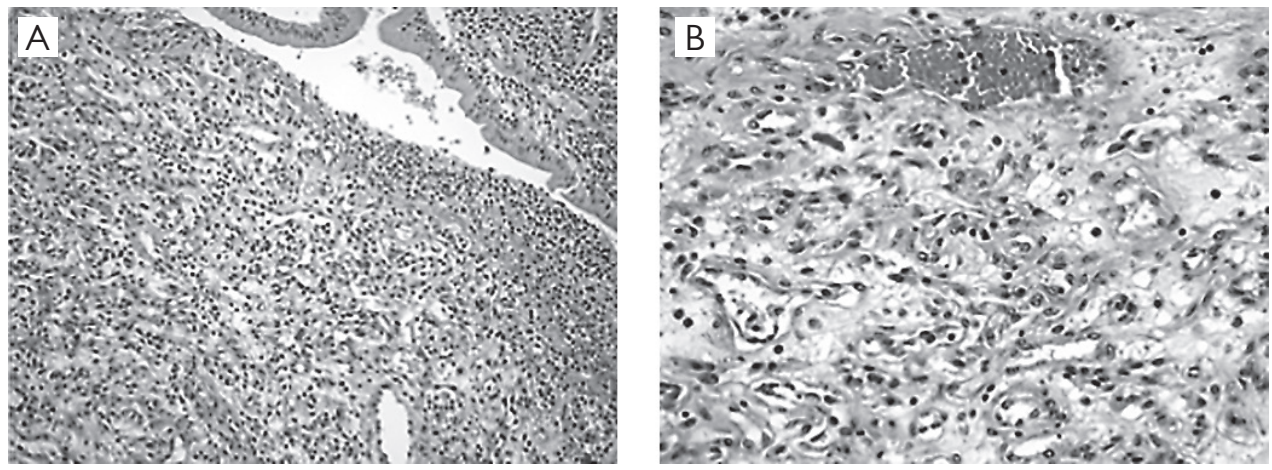

Figure 1 - Microscopic examination of the resected duodenum and pancreas.

Microscopic examination of the resected duodenum and pancreas show clear cell carcinoma compatible with metastasis from renal cell carcinoma. Tumour cells are large, with abundant eosinophilic cytoplasm. Their appearance is highly suggestive of renal cell carcinoma cells (hematoxylin-eosin, a: $\times 200, b: \times 400$ ).
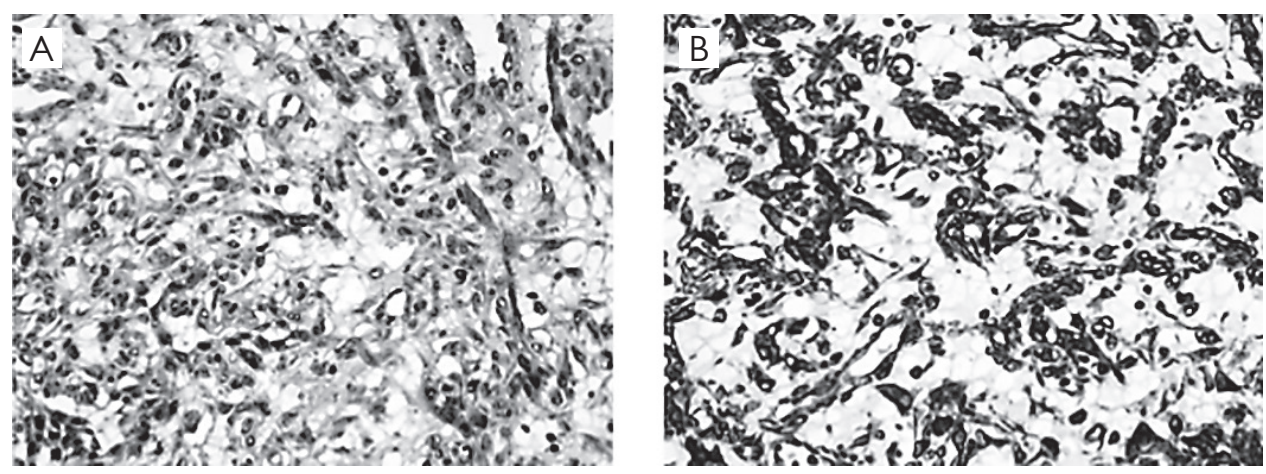

Figure 2 - Histological section of the ampullary wall.

The diagnosis renal cell carcinoma metastasis is confirmed by the immunohistochemical detection of cytokeratin (a) and vimentin (b).

were free. The postoperative course was uneventful, and there were no signs of local and systemic recurrence. Patient died from cardiovascular disease 14 years.

\section{Discussion}

Distant metastases are dominating the course of renal cell carcinoma in more than $60 \%$ of patients (Pavlakis et al., 2004; Wente et al., 2005). Renal cell carcinoma may spread to any organ, the most common sites for metastases being the lymph nodes, lung, bone, skin, adrenal glands, liver, opposite kidney, brain (Jacobs et al., 2013) and - to a smaller extent - pancreas (Hirota et al., 1996; Hashimoto et al., 2001; Wente et al., 2005), whereas ampullary metastases are reported to be extremely rare (Pavlakis et al., 2004; Merino et al., 2005; Ogiso et al., 2005; Zhao 
et al., 2012; Smigielski et al., 2013). To our knowledge, there are only 12 cases of ampulla of Vater metastases from renal cell carcinoma reported in the English, German, Japanese, and Scandinavian literature (Leslie et al., 1996; Janzen et al., 1998; Merino et al., 2005; Ogiso et al., 2005; Chowdhury et al., 2014; Cheong et al., 2018; Sarocchi et al., 2018).

Le Borgne et al. (2000) provided a detailed analysis of symptoms and signs of metastatic tumours of the ampullary region. The most common symptoms of presentation were jaundice, acute pancreatitis related to pancreatic duct obstruction, and upper gastrointestinal tract bleeding. In the majority of patients, it is not possible to differentiate clinically metastatic lesions in the papilla from a primary tumour, although this would obviously be of importance in patients with a history of malignancy.

It is of certain interest to find that the mean time interval between surgery for a primary tumour and the diagnosis of a metastatic ampullary tumour was 88 months in the study of Le Borgne et al. (2000). Regarding the prognosis, the longer the interval between the primary tumour resection and the diagnosis of metastases the longer the disease-specific survival may be expected (Hirota et al., 1996; van der Poel et al., 1999). The efficacy of operative resection of localized renal carcinoma metastatic lesions in the liver, lungs, and brain is well established and an aggressive approach is suggested (Nakeeb et al., 1995; Leslie et al., 1996; Le Borgne et al., 2000). A series from The Johns Hopkins' has also shown favourable results regarding survival rate of the patients, operated on because of primary periampullary carcinoma followed by radically intended metastasectomy (Nakeeb et al., 1995). Curative pancreatectomy resulted in zero mortality and an overall survival of $35 \%$ at 2 years and $17 \%$ at 5 years according to Le Borgne et al. (2000) report. This means that surgery is indicated not only as a life-saving procedure to manage metastasis-related complications, e.g. intestinal obstruction, gastrointestinal bleeding, and jaundice, but also as an elective procedure with the aim to resect the metastatic lesion with the aim of cure (Haidong et al., 2014). However, the majority of patients with renal cell carcinoma metastases eventually require complementary systemic therapy, because they often already have metastases in other locations not seldom occult until carefully looked for. Therefore, treatment in such cases must be individually tailored.

In conclusion, we report a case of ampulla of Vater metastasis from renal cell carcinoma. Severe transient epigastric pain, possibly due to outflow obstruction of the pancreatic duct, and transient jaundice were the major manifestation of the metastasis. The patient was successfully treated by curative pancreatoduodenectomy. In the absence of generalized metastatic disease, it is obvious that pancreatoduodenectomy can provide long-term survival in selected cases with solitary metastasis. Favourable long-term survival rates suggest that these patients should be considered candidates for pancreatoduodenectomy as long as experienced pancreatic surgeon is available and no other metastases are found. 


\section{References}

Cheong, D., Rho, S. Y., Kim, J. H., Kang, C. M., Lee, W. J. (2018) Laparoscopic pancreaticoduodenectomy for renal cell carcinoma metastasized to ampulla of Vater: A case report and literature review. Ann. Hepatobiliary Pancreat. Surg. 22(1), 83-89.

Chowdhury, S. D., Masih, D., Chawla, G., Pal, S., Kurien, R. T., Augustine, J. (2014) Metastasis of renal cell carcinoma to the duodenal papilla. Indian J. Gastroenterol. 33(5), 493-494.

Haidong, W., Jianwei, W., Guizhong, L., Ning, L., Feng, H., Libo, M. (2014) Ampullary tumor caused by metastatic renal cell carcinoma and literature review. Urol. J. 11(2), 1504-1507.

Hashimoto, M., Miura, Y., Matsuda, M., Watanabe, G. (2001) Concomitant duodenal and pancreatic metastases from renal cell carcinoma: report of a case. Surg. Today 31(2), 180-183.

Hirota, T., Tomida, T., Iwasa, M., Takahashi, K., Kaneda, M., Tamaki, H. (1996) Solitary pancreatic metastasis occurring eight years after nephrectomy for renal cell carcinoma. A case report and surgical review. Int. J. Pancreatol. 19(2), 145-153.

Jacobs, C., Kim, D. W., Straka, C., Timmerman, R. D., Brugarolas, J. (2013) Prolonged survival of a patient with papillary renal cell carcinoma and brain metastases using pazopanib. J. Clin. Oncol. 31(7), e114-e117.

Janzen, R. M., Ramj, A. S., Flint, J. D., Scudamore, C. H., Yoshida, E. M. (1998) Obscure gastrointestinal bleeding from an ampullary tumour in a patient with a remote history of renal cell carcinoma: a diagnostic conundrum. Can. J. Gastroenterol. 12(1), 75-78.

Karakatsanis, A., Vezakis, A., Fragulidis, G., Staikou, C., Carvounis, E. E., Polydorou, A. (2013) Obstructive jaundice due to ampullary metastasis of renal cell carcinoma. World J. Surg. Oncol. 11, 262.

Le Borgne, J., Partensky, C., Glemain, P., Dupas, B., de Kerviller, B. (2000) Pancreaticoduodenectomy for metastatic ampullary and pancreatic tumors. Hepatogastroenterology 47(32), 540-544.

Leslie, K. A., Tsao, J. I., Rossi, R. L., Braasch, J. W. (1996) Metastatic renal cell carcinoma to ampulla of Vater: an unusual lesion amenable to surgical resection. Surgery 119(3), 349-351.

Merino, C., Moles, J. R., Rodrigo, A., Ferrando, J., Garcia, J., Primo, J., Albert, A., Arago, M., Serra, B., Amoros, I. (2005) Uncommon etiology of gastrointestinal bleeding: duodenal metastases from renal cell carcinoma. Gastroenterol. Hepatol. 28(4), 221-224. (in Spanish)

Nakeeb, A., Lillemoe, K. D., Cameron, J. L. (1995) The role of pancreaticoduodenectomy for locally recurrent or metastatic carcinoma to the periampullary region. J. Am. Coll. Surg. 180(2), 188-192.

Ogiso, S., Maeno, A., Nagahama, K., Nakamura, K., Okuno, H. (2005) Small intestinal metastases from renal cell carcinoma: a case report and literature review. Hinyokika Kiyo 51(1), 13-16. (in Japanese)

Pavlakis, G. M., Sakorafas, G. H., Anagnostopoulos, G. K. (2004) Intestinal metastases from renal cell carcinoma: a rare cause of intestinal obstruction and bleeding. Mt. Sinai J. Med. 71(2), 127-130.

Sarocchi, F., Gilg, M. M., Schreiber, F., Langner, C. (2018) Secondary tumours of the ampulla of Vater: Case report and review of the literature. Mol. Clin. Oncol. 8(2), 274-280.

Smigielski, J., Piskorz, L., Wawrzycki, M., Jablonski, S., Brocki, M. (2013) A case of upper gastrointestinal acute bleeding as a complication of renal carcinoma metastases to the papilla Vateri. Contemp. Oncol. (Pozn.) 17(5), 470-472.

van der Poel, H. G., Roukema, J. A., Horenblas, S., van Geel, A. N., Debruyne, F. M. (1999) Metastasectomy in renal cell carcinoma: A multicenter retrospective analysis. Eur. Urol. 35(3), 197-203.

Wente, M. N., Kleeff, J., Esposito, I., Hartel, M., Muller, M. W., Frohlich, B. E., Buchler, M. W., Friess, H. (2005) Renal cancer cell metastasis into the pancreas: a single-center experience and overview of the literature. Pancreas 30(3), 218-222.

Zhao, H., Han, K., Li, J., Liang, P., Zuo, G., Zhang, Y., Li, H. (2012) A case of wedge resection of duodenum for massive gastrointestinal bleeding due to duodenal metastasis by renal cell carcinoma. World J. Surg. Oncol. 10, 199. 